\title{
INVESTIGACIÓN BIOGRÁFICA Y AUTOBIOGRÁFICA EN EDUCACIÓN EN AMÉRICA LATINA
}

\section{Presentación}

Los artículos sometidos para la composición del presente dossier representan una mirada plural acerca de la aproximación conceptual, metodológica y experiencial desarrollada desde las ciencias sociales y la investigación biográfico-narrativa en educación de América Latina aplicada tanto en ámbitos formales como no formales. Los ejes temáticos, a los que corresponden distintos modos de abordar, revelan la actualidad del campo distribuidos en tres agrupamientos principales: estado del conocimiento o estado del arte en determinados países durante la última década; enfoques y métodos de investigación biográfico-narrativa en la educación; instituciones y sujetos, enlaces biográficos.

En la producción de América Latina es notable la interconexión de los campos de conocimiento; deja ver en un campo biográfico fértil que recoge las variadas deliberaciones provenientes de la literatura inglesa, francesa, alemana e italiana, junto con la proyección creciente de la lengua portuguesa. En contraste, es débil el eco de otras zonas geográficas como Asia o África, probablemente a causa de circuitos limitados de circulación más que de producción. El enfoque biográfico en las narrativas de educación ciertamente se remonta pocos decenios atrás, si tomamos en cuenta la sistematización de argumentaciones y enfoques paradigmáticos; empero no deja de hundir sus raíces en una tradición de emergencias y rupturas en el universo de las ciencias sociales.

Schulze (1993) ${ }^{1}$ planteó por lo menos dos

1 SCHÜLZE, Theodor. Pedagogía con orientación biográfica. Educación. Colección semestral de aportaciones alemanas recientes en las ciencias pedagógicas. Instituto de colaboración científica Tubingen. Vol. 48, p. sendas de desarrollo de lo biográfico: hacia la investigación en educación y hacia las deliberaciones encaminadas a la elucidación de una teoría de la formación vinculada a tradiciones teóricas de las ciencias sociales. En cada una indicó algunas veredas a donde habría de conducir el tema de lo biográfico. Con el paso del tiempo, la reflexión sobre lo biográfico en nuestro continente ha ampliado su ámbito de acción y ha ensanchado las tradiciones en las que abreva América Latina.

El campo actual de la investigación biográfico-narrativa en educación, sin perjuicio de reconocer distintas filiaciones disciplinares, usufructúa conceptos básicos con los que aborda el análisis de la condición biográfica en el mundo contemporáneo. Participamos de un "hecho antropológico" (DELORY-MOMBERGER, 2009, 2015)² reflejado en los pequeños detalles del mundo de relaciones que se tejen entre el individuo y las representaciones de sí y de los otros, en las relaciones de trabajo y en la escuela, en el núcleo familiar, en la amistad y el amor.

La trascendencia del diálogo planteado entre la tradición de las ciencias sociales y la condición biográfica actual deja ver que

\section{8-100, 1993.}

2 DELORY-MOMBERGER, Christine. La condition biographique. Essais sur le récit de soi dans la modernité avancée. París: Téraedre, 2009. [Hay traducción española: Delory-Momberger, C. La condición biográfica. Ensayos sobre el relato de sí en la modernidad avanzada. Traducción Miguel Orlando Betancur. Medellín: Universidad de Antioquia, 2015]. DELORY-MOMBERGER, Christine. "Ser alumno: entre ritualizaciones escolares y la construcción de sí". En Murillo, Gabriel Jaime (coord.) Narrativas de experiencia en educación y pedagogía de la memoria. Buenos Aires: Ed. FFyL-UBA/ CLACSO/Universidad de Antioquia, 2015, p. 207-214. 
[...] los asuntos biográficos, bajo cualquier denominación, son las temáticas de las ciencias sociales en su conjunto o, dicho de otra manera, la aproximación biográfica es un punto de intersección de los campos de conocimiento, a la vez que mantiene disposiciones epistemológicas y teórico-metodológicas que lo constituyen como campo específico y consolidado de investigación [...] lo biográfico se ha convertido en el epítome de las ciencias sociales modernas y del saber educativo en general. (SOUZA, SERRANO y RAMOS, 2014, p. 684) ${ }^{3}$

El enfoque biográfico en educación está en una deuda con los conceptos, métodos y variados objetos de estudio puestos en juego en los trabajos de la antropología, la sociología, la historia, las artes y la literatura. Sobre este telón de fondo se ha desplegado a lo largo del último siglo un renacer de la narrativa de la mano del retorno del sujeto, ya sea amplificada en las voces de los inmigrantes europeos en América, y más recientemente de africanos, asiáticos y latinos en Europa, o de centroamericanos que traspasan la frontera de Estados Unidos o del éxodo de venezolanos hacia todas partes; y de las víctimas y soldados que retornan de la guerra, o en las voces del pasado o de los excluidos y marginados y extranjeros en las sociedades posindustriales y en las poscoloniales, que hizo posible la elevación de los testimonios vivos a la categoría de documentos de la cultura.

El presente dossier constituye un eslabón más en la cadena forjada desde los albores del siglo XXI con intereses en una reflexión permanente sobre la dinámica de las producciones teóricas y del campo de aplicación de las narrativas biográficas en educación. Justo es decirlo que a la continuidad de los CIPA (Congresso International de Pesquisa Autobiográfica) en Brasil, complementada a su vez con la

3 SOUZA, E.C, SERRANO, J.A. Y RAMOS, J.M (2014). Autobiografía y educación. Tradiciones, diálogos y metodologías. Revista Mexicana de Investigación Educativa, 62.3, 2014, Ciudad de México, p. 683-694. fundación de BIOgraph (Asociación Brasileña de Investigación (Auto)biográfica), se suma la realización de eventos académicos de intercambio en otros países tales como: Seminario Internacional Documentación Narrativa de Experiencias Pedagógicas, Argentina; Simposio Internacional Narrativas en Educación, Colombia; también en Chile, Costa Rica, Puerto Rico y México. En este último -a modo de estado de conocimiento- destaca la publicación del número $62-\mathrm{y}$ los dos números subsecuentes- de la Revista Mexicana de Investigación Educativa con una sección temática dedicada a "Autobiografía y educación: tradiciones, diálogos y metodologías" en donde la idea de inventario de lo producido se pone a prueba.

El primer eje del dossier da cuenta de los modos de producción de las narrativas biográficas en educación, a través de una analítica desarrollada explícitamente en el caso de Argentina, Brasil y México, expresada tácitamente en los demás países considerados aquí. Elaborar estados de conocimiento es una forma de estar al día en los asuntos de las temáticas, las metodologías, los autores que forman el hit parade del campo biográfico narrativo.

En nuestro andar hemos visto el pasaje de variadas denominaciones para el tema de la justificación de la investigación. Hace décadas se hablaba de la búsqueda de "la tradición de lo que se ha investigado sobre el tema", luego se pasó a la noción de "estado de la cuestión", hasta llegar al "estado de conocimiento" y culminar con la idea de "estado de arte". Por la interconexión de ideas y textos, sin duda el ámbito de la investigación es cada vez más canónico. Normatividad en uso que deja sus ventajas para elaborar estados de conocimiento: inscribir las ideas propias en tradiciones o preguntas previamente realizadas para profundizar, rellenar lagunas, o buscar nuevas vetas ante preguntas clásicas con nuevos paradigmas. A pesar de la exigencia institucional 
de la elaboración de estados de conocimiento y de las ventajas en el proceso investigativo, poca reflexión sobre su confección se ha realizado en la literatura. Como advierte Becher, la construcción del conocimiento "contempla varios aspectos diferentes: una comunidad, una red de comunicaciones, una tradición, un conjunto particular de valores y creencias, un dominio, una modalidad de investigación y una estructura conceptual" (2001, p. 38)

El segundo eje asumido por los autores que integran el dossier se refiere a los aspectos metodológicos ligados a las reflexiones sobre el estatuto del campo. La búsqueda de nuevas vetas para producir datos está ligada a la tradición, de la cual somos herederos, que se remonta al libro de Thomas y Znaniecki [El campesino polaco en Europa y en América.1918-1920] que es "La premiere utilization sociologique de documents d'ordre biographique et autobiographique" (DUBAR y NICOURD, 2017, p. 7) $)^{5}$. En este punto es inevitable reconocer la huella perdurable que ha dejado la Escuela de Chicago, gracias a la cual las cartas dejaron de ser cartas, el diario dejó de ser diario, para pasar a la categoría metodológica de “documentos personales" (PLUMMER, 1989) ${ }^{6}$. Categoría metodológica que se suma a la variabilidad de estrategias al uso en las investigaciones cualitativas, como parte de la caja de herramientas que incorpora entrevistas a profundidad, la observación participante y los instrumentos tecnológicos que van desde la grabación sonora, la fotografia, hasta el uso de los teléfonos inteligentes para nutrir las reflexiones sobre la vida.

4 BECHER, Tony. Tribus y territorios académicos. La indagación intelectual de las disciplinas académicas. Traducción de Andrea Menegotto. Barcelona: Gedisa, 2011.

5 DUBAR, Claude y NICOURD, Sandrine. Les biographies en sociologie. Paris: Éditions La Découverte, 2017.

6 PLUMMER, Ken. Los documentos personales. Introducción a los problemas y la bibliografía del método humanista. Traducción Julio Velasco Cobelo. Madrid: Siglo XXI, 1989.
El tercer eje temático se ocupa de la experiencia escolar como objeto de estudio que atraviesa variados programas de formación, investigación y extensión. Por supuesto, un enunciado de $\tan$ amplios alcances permite cubrir las trayectorias de una persona a lo largo de una vida, la trayectoria biográfica de un profesor, la identidad profesional docente, la cultura escolar, en fin, los vínculos entre individuo e institución. El tema individuo-institución tiene sus raíces en la propuesta de la sociología fenomenológica de Berger y Luckman que afirma: "El universo simbólico aporta el orden para la aprehensión subjetiva de la experiencia biográfica" (1983, p. 127)7 , para explicar los trasvases que existen entre la vida individual y el movimiento social. Si bien la institución tiene efectos instituidos, el individuo hace institución a través de sus procesos instituyentes. De ello se desprende que la institución no es una entelequia; el sujeto está implicado en ella y no se entienden uno sin la otra.

En ciencias sociales encontramos al menos cuatro caminos para dar cuenta del vínculo individuo-institución: el análisis institucional, de tradición francesa; el enfoque sociológico del estudio de las generaciones, la sociología de las profesiones y la sociología de las instituciones. El primero busca intervenir en las instituciones y de ahí analizar las prácticas de sus integrantes. El segundo explica los vínculos individuo-grupo en la conformación del espíritu de una época; entramado que da lugar al estudio de la relación entre individuo y clase, las generaciones y los modos de socialización que ponen el acento en la agencia del individuo. Como señala Villegas $(1998)^{8}$, las relaciones espacio temporales conforman la biografia

7 BERGER, Peter y LUCKMAN, Thomas. La construcción social de la realidad. Traducción de Silvia Zuleta. Buenos Aires: Amorrortu, 1983.

8 VILLEGAS, Francisco. Los profetas y el mesías: Lukás y Ortega como precursores de Heidegger en el Zeigest de la modernidad (1900-1929). Ciudad de México: Fondo de Cultura Económica, 1998. 
de los particulares, tiñen su obra, más allá de las relaciones cara a cara, lo que está en juego es la afinidad de percibir la realidad.

El tercer camino está orientado a elucidar el entramado entre ocupación y empleo y las formas de institucionalización de las prácticas de nuevas formas de hacer (BALLESTEROS, 2007)9. El cuarto, nutrido de las preocupaciones sustantivas de las grandes teorías sociológicas $-y$ reformuladas con la aparición de la nueva sociología de la educación en los años setenta del siglo pasado-, investiga los procesos sobre los cuales se construye la subjetividad en la época moderna. (Para el caso, revisar las elucubraciones de Álvarez-Uría y Varela, 2009) $)^{10}$.

Algunas de estas vetas fueron retomadas en los textos que presentamos en el dossier. El primero de ellos, a modo de inventariado del campo, Documentar la experiencia biográfica y pedagógica. La investigación narrativa y (auto)biográfica en educación en Argentina de Daniel Hugo Suárez y Paula Valeria Dávila, propone deslindar, por un lado, los trabajos de investigación educativa que hacen uso de las narrativas como método y, por otro lado, la investigación narrativa autobiográfica inscrita en el campo de la pedagogía. La exposición plantea la paradoja según la cual la cartografía muestra un campo fértil para el pensamiento y la acción pedagógica, aun cuando sean cortos sus alcances dado el número de grupos académicos que se reclaman del enfoque biográfico narrativo.

Más adelante, José Antonio Serrano y Juan Mario Ramos en Estudios biográficos en instituciones educativas en México, retoman la tradición de la elaboración de estados de conocimiento. De la prolija bibliografía confecciona-

9 BALLESTEROS, Arturo. Max Weber y la sociología de las profesiones. Ciudad de México: UPN, 2007.

10 ÁLVAREZ-URÍA, Fernando y VARELA, Julia. Sociologia de las instituciones. Bases sociales y culturales de la conducta. Madrid: Morata, 2009. da en los últimos diez años en el país, retoman los textos publicados por connacionales en revistas, capítulos de libros y libros. A partir de dar la voz a los autores, exponen la traducción que buena parte de ellos hacen de la noción de trayectoria en la comprensión de la experiencia de los agentes sociales (alumnos, maestros, personal directivo y de apoyo) al dar vida a las instituciones educativas. Los autores muestran la diversificación de los autores y perspectivas y el transvase de ideas sobre el campo biográfico-narrativo.

Luego, el texto de Andrés Klaus Runge y Gabriel Jaime Murillo, titulado Profesión maestro y cultura escolar: relatos de experiencia, pone en escena un diálogo a muchas voces desarrollado en torno a los fundamentos conceptuales y metodológicos de un proyecto de investigación sobre la vida profesional de los maestros rurales en Antioquia (Colombia). "Del diálogo hacen parte alrededor de dos centenares de maestros rurales, junto con los profesores investigadores de nueve universidades de la región", quienes previamente participaron de un seminario de formación basado en la investigación biográfico-narrativa en educación. En la primera fase del proyecto los maestros produjeron narrativas de "memoria escolar entre la guerra y la paz, mientras en la segunda fase, todavía en marcha, la atención se centra en los relatos de experiencia de maestros en tiempos de incertidumbre", en el marco de un estudio cualitativo sobre la cultura escolar.

Por su parte, Ricia Anne Chansky, Edward Contreras Santiago, Fernando Correa González, Marci Denesiuk, Jocelyn Géliga Vargas y Catherine Mazak Chansky estudian la vinculación entre la educación y la justicia social en el trabajo titulado I is for Agency: Education, Social Justice, and Auto/Biographical Practices (Sou de agência: educação, justiça social e práticas auto/biográficas). Se trata de un ensayo que interconecta las micronarrativas surgidas 
de las prácticas autobiográficas que fueron incorporadas en el currículo del departamento de inglés en la sede Mayagüez de la Universidad de Puerto Rico, con una reflexión acerca de los medios de fomentar la reflexividad de sí, el empowerment, en las aulas y en el resto de la sociedad en tiempos de crisis. La crisis refiere a la vez a la depresión económica que atraviesa desde tiempo atrás la isla, sometida al férreo control fiscal del congreso de USA y, sobre todo, a la crisis humanitaria ocasionada por la devastación general que dejó el huracán del 20 de septiembre de 2017.

Un ejemplo acerca del tratamiento del vínculo existente entre institución e individuos, se encuentra en el artículo de María Cristina Sarasa Narrativas vitales en las socialidades del currículo universitario para la formación del profesorado, que considera el currículo como el lugar de negociación de significados, con efectos dispares en las vidas de los actores del proceso de formación del profesorado en inglés. El artículo deja ver los efectos de la socialización, la configuración de lo público y lo privado en la trama institucional de formar docentes en contextos universitarios.

El artículo Trajetórias de professoras e histórias improváveis de sucesso na escola sob o olhar da memória (1870-1985) de Paula Vicentini, Rita de Cássia Gallego y Vivian Batista da Silva expone el efecto instituyente de tres profesoras en instituciones educativas de la ciudad de Niterói (Brasil) entre 1870 y 1985. Las vidas de estas mujeres, que dejaron huella en la dirección de centros educativos y en la escritura de textos sobre Pedagogía y Didáctica, son reconstruidas a partir de la exploración de variadas fuentes orales y escritas, relatos autobiográficos de las protagonistas, informes de inspectores escolares, notas de prensa y entrevistas, lo cual da testimonio del valor de los documentos personales en los estudios históricos y sociales en educación.
El artículo de Martha Cecilia Herrera y Vladimir Olaya, Relatos de vida: una puerta de entrada a las subjetividades de los maestros en Colombia, describe un trayecto de biografización de dos maestras llevado a cabo a través de múltiples instancias como talleres, entrevistas, diarios personales, fotografias, música y literatura. En el curso del trayecto, los sujetos van trenzando diversas discursividades, temporalidades y espacialidades, así como las maneras como estos procesos alientan la transformación de prácticas pedagógicas y permiten avizorar modos de resignificación del pasado violento.

A propósito de la relación entre sujeto y constitución de los campos de conocimiento, Rosa María Torres en Linaje, compromisos personales e institucionales en la filosofia hispanoamericana. Biografía de Fernando Salmerón Roiz, reflexiona sobre las relaciones entre continuidad y discontinuidad en el campo y el lugar de la dimensión existencial y racional en las biografias intelectuales de los individuos. Con base en las vivencias personales, el sentido de la adscripción académica y sus efectos en la afirmación de la institucionalidad, este perfil biográfico muestra el lugar de la "grafia" como huella de la producción intelectual que crea simultáneamente filiaciones personales e institucionales.

En el ámbito de la formación continua, Ruslane Marcelino de Mello Campo Novais y Valdete Côco, en Abordagem biográfica: a dimensão da vida presente nos enunciados de pesquisa em educação, presentan el delineamiento metodológico elaborado para una investigación narrativa en el campo de la formación continuada en un contexto de educación infantil. Los resultados evidencian explícitamente una articulación entre el yo personal y el yo profesional, que sirve de fundamento para un diálogo sobre la formación continuada y la actuación docente. 
El tema de lo metodológico resalta en el trabajo de Guilherme do Val Toledo Prado, Renata Barroso Siqueira Frauendorf y Grace Carolina Chavez Buldrin Chautz, Inventário de pesquisa: uma possibilidade de organização de dados da investigação. Con base en una lectura de tesis y disertaciones elaboradas en un decenio, que totalizan 70 producciones del grupo, se da curso a un ejercicio de inventariar dicho acervo informativo como un procedimiento metodológico que invita al investigador a revisar su práctica pedagógica, clasificando e interpretando los documentos catalogados, al mismo tiempo de tomar conciencia del saber de experiencia que se ha ido construyendo a lo largo de la vida.

Finaliza el dossier con el texto de Diego Leandro Marín Ossa, que lleva por título Coordenadas del video autobiográfico. El relato de la experiencia de vida mediada, mediática y mediatizada. A partir de establecer la distinción entre video autobiografía y video autobiográfico, se apela al entendimiento de una autobiografía en donde no se habla ya de autor y narrador, se habla de autobiógrafo, quien adquiere y desarrolla las habilidades y la con- ciencia de sí por medio del relatar y el reflexionar sobre lo vivido, lo recordado y lo relatado. A continuación, se exponen ideas relacionadas con la experiencia mediatizada desde el aprendizaje, los medios de comunicación, la sociología, y el lenguaje, que sustentan un cuestionamiento acerca del modo como la interacción con las pantallas y las imágenes modifican nuestra mirada, nuestro pensamiento y aun nuestra propia existencia.

Estamos seguros de que este es un primer recuento de lo que se produce en América Latina. No dejará de ser una puerta de entrada para las nuevas deliberaciones y los retos de experimentación en el campo biográfico narrativo que se avecinan a futuro.

Ciudad de México, Medellín, 20 de agosto de 2018

José Antonio Serrano Castañeda Universidad Pedagógica Nacional - México Gabriel Jaime Murillo Arango Universidad de Antioquia - Colombia 